PROCEEDINGS OF THE

AMERICAN MATHEMATICAL SOCIETY

Volume 140, Number 1, January 2012, Pages 135-146

S 0002-9939(2011)11273-5

Article electronically published on July 13, 2011

\title{
SEPARATING INVARIANTS FOR THE BASIC $\mathbb{G}_{a}$-ACTIONS
}

\author{
JONATHAN ELMER AND MARTIN KOHLS
}

(Communicated by Harm Derksen)

\begin{abstract}
We explicitly construct a finite set of separating invariants for the basic $\mathbb{G}_{a}$-actions. These are the finite dimensional indecomposable rational linear representations of the additive group $\mathbb{G}_{a}$ of a field of characteristic zero, and their invariants are the kernel of the Weitzenböck derivation $D_{n}=x_{0} \frac{\partial}{\partial x_{1}}+$ $\ldots+x_{n-1} \frac{\partial}{\partial x_{n}}$.
\end{abstract}

\section{INTRODUCTION}

A great many mathematical problems are special cases of the following: let $K$ be a field of arbitrary characterstic and let $G$ be any group. Suppose $G$ acts on the $K$-vector space $V$ and that $v$ and $w$ are points of $V$. Is there a $g \in G$ satisfying $g v=w$ ? In other words, are $v$ and $w$ contained in the same $G$-orbit? Important examples include the case where $G=\mathrm{GL}_{n}(K)$ acts on the vector space $V$ of $n \times n$ matrices by conjugation, and the case where $G=\mathrm{SL}_{n}(K)$ acts on the space $V$ of binary forms of degree $n$. The classical approach to these problems is to construct "invariant polynomials". These are polynomial functions $f: V \rightarrow K$ which satisfy $f(v)=f(g v)$ for all $g \in G$ and $v \in V$, and so are constant on $G$ orbits. In fact, one can define an action of $G$ on the set of polynomial functions $K[V]$ via $(g \cdot f)(v):=f\left(g^{-1} v\right)$ for which the invariant polynomials are the fixed points, $K[V]^{G}$, and these form a subalgebra of $K[V]$ called the algebra of invariants. Ideally, one would like to find a complete set of algebra generators of $K[V]^{G}$, then use this set to distinguish as many orbits as possible.

This approach is not without its difficulties. For instance, it is not always possible to distinguish all the orbits using invariant polynomials. As an example, consider once more the case where $\mathrm{GL}_{n}(K)$ acts on the vector space of $n \times n$ matrices over a field $K$ by conjugation. Provided $K$ is an infinite field, the invariants are generated by the coefficients of the characteristic polynomial [5. Example 2.1.3], but it is well known that a pair of matrices with the same characteristic polynomial are not necessarily conjugate. More problematically, if $G$ is not reductive, then the algebra $K[V]^{G}$ may not even be finitely generated. Even if it is, finding a set of generators can be a very difficult problem. If, however, one is only interested in invariants from the point of view of separating orbits, then finding a complete set of generators is

Received by the editors November 12, 2010.

2010 Mathematics Subject Classification. Primary 13A50, 13N15.

Key words and phrases. Invariant theory, separating invariants, binary forms, locally nilpotent derivations, basic $\mathbb{G}_{a}$-actions, generalized hypergeometric series.

(C)2011 American Mathematical Society 
not always necessary. It is perhaps surprising, then, that Derksen and Kemper made the following definition [5, Definition 2.3.8] as recently as 2002.

Definition 1.1. A separating set for the ring of invariants $K[V]^{G}$ is a subset $S \subseteq K[V]^{G}$ with the following property: given $v, w \in V$, if there exists an invariant $f$ satisfying $f(v) \neq f(w)$, then there also exists $s \in S$ satisfying $s(v) \neq s(w)$.

There are many instances in which separating sets can be seen to have "nicer" properties than generating sets. For example, it is well known that if $G$ is finite and the characteristic of $K$ does not divide $|G|$, then $K[V]^{G}$ is generated by elements of degree $\leq|G|$, see [10, 11, but this is not necessarily true in the modular case (i.e. when the characteristic of $K$ divides $|G|$ ). On the other hand, the analogue for separating invariants holds in arbitary characteristic [5, Theorem 3.9.13]. Meanwhile, even if $K[V]^{G}$ is not finitely generated, it is guaranteed to contain a finite separating set [5. Theorem 2.3.15]. The existence proof is nonconstructive, which raises the question how to actually construct separating sets. Kemper [15] gives an algorithm for reductive groups, but using Gröbner bases it is only effective for "small" cases. An example of a finite separating set for a non-finitely generated invariant ring is given in 8]. For finite groups, a separating set can always be obtained as the coefficients of a rather large polynomial [5, Theorem 3.9.13]. With refined methods, "nicer" separating sets have been obtained for several classes of finite groups and representations; see for example 20]. This paper goes in the same direction: for the basic actions of the additive group in characteristic zero, we present a rather small separating set. See also [6, 7, 9, 16, for a small selection of other recent publications in the area.

From this point onwards, $\mathbb{k}$ denotes a field of characterstic zero. In this article we will concentrate on linear actions of the additive group $\mathbb{G}_{a}$ of the ground field $\mathbb{k}$. The finite dimensional indecomposable rational linear representations of $\mathbb{G}_{a}$ are called the basic $\mathbb{G}_{a}$-actions. There is one such action in each dimension, and these are described below:

Definition 1.2. Let $X_{n}:=\left\langle x_{0}, \ldots, x_{n}\right\rangle_{\mathbb{k}}$ be a vector space of dimension $n+1$. Then $\mathbb{G}_{a}$ is said to act basically on $X_{n}$ (with respect to the given basis) if the action of $\mathbb{G}_{a}$ on $X_{n}$ is given by the formula

$$
a * x_{i}=\sum_{j=0}^{i} \frac{a^{j}}{j !} x_{i-j}, \quad \text { for all } a \in \mathbb{G}_{a}, i=0, \ldots, n .
$$

Note the isomorphisms $X_{n} \cong X_{n}^{*} \cong S^{n}\left(X_{1}\right)$ for all $n$, where $S^{n}$ denotes the $n$th symmetric power. Let $\left\{x_{0}, x_{1}, \ldots, x_{n}\right\}$ be the set of coordinate functions on an $(n+1)$-dimensional vector space $V_{n}$, so we consider $X_{n}=V_{n}^{*}$. As $\mathbb{k}$ is infinite, $\mathbb{k}\left[V_{n}\right]$ can be viewed as the polynomial ring $R_{n}:=S\left(X_{n}\right)=\mathbb{k}\left[x_{0}, x_{1}, \ldots, x_{n}\right]$. If $\mathbb{G}_{a}$ acts basically on $X_{n}$, one can then check that the induced action of $\mathbb{G}_{a}$ on $R_{n}$ is given by the formula

$$
a * f=\exp \left(a D_{n}\right) f \quad \text { for all } a \in \mathbb{G}_{a}, f \in R_{n},
$$

where $D_{n}$ is the Weitzenböck derivation

$$
D_{n}:=x_{0} \frac{\partial}{\partial x_{1}}+\cdots+x_{n-1} \frac{\partial}{\partial x_{n}} \quad \text { on } R_{n} .
$$

Furthermore, the algebra of invariants $\mathbb{k}\left[V_{n}\right]^{\mathbb{G}_{a}}$ is precisely the kernel of $D_{n}$. We denote this by $A_{n}$. The algebras $A_{n}$ have been objects of intensive study for well 
over a hundred years, owing to their connection with the classical invariants and covariants of binary forms. While they are known to be finitely generated by the Maurer-Weitzenböck Theorem [24, the number of generators appears to increase rapidly with dimension, and explicit generating sets are (reliably!) known only for $n \leq 7$ (see also the table in section 2). In this article, we shall instead construct explicit separating sets for all values of $n$.

This article is organised as follows. In Section 2 we state our main results and explain briefly the connection between the algebras $A_{n}$ and the covariants of binary forms. In Section 3 we prove a crucial lemma on the radical of the Hilbert ideal of $A_{n}$ which may be of independent interest. Section 4 contains the main body of the proof of our result, while Section 5 is devoted to the proof of a technical lemma which is required in order to construct a separating set for $A_{n}$ when $n \equiv 0 \bmod 4$.

\section{BACKGROUND AND STATEMENT OF RESUlts}

Let $U_{n}$ denote the $\mathbb{k}$-vector space of binary forms of degree $n$, which are homogeneous polynomials of the form $\sum_{i=0}^{n} a_{i} X^{i} Y^{n-i}$ in the variables $X$ and $Y, a_{i} \in \mathbb{k}$. This is a vector space of dimension $n+1$ with basis the set of monomials in $X$ and $Y$ of degree $n$. The natural action of the group $G:=\mathrm{SL}_{2}(\mathbb{k})$ on a two dimensional vector space with basis $\{X, Y\}$ induces an action of $G$ on the vector space $U_{n}$. Classically speaking, an invariant is a polynomial in the coefficients $a_{i}$ which is unchanged under the action of $G$, in modern notation, an element of $\mathbb{k}\left[U_{n}\right]^{G}$. Note that the additive group $\mathbb{G}_{a}$ is embedded in $G$ as the subgroup of matrices of the form $\left(\begin{array}{cc}1 & * \\ 0 & 1\end{array}\right)$, and the subgroup $\mathbb{G}_{a}$ acts basically on $U_{n}$ (with respect to the basis $\left.\left\{\frac{1}{k !} X^{n-k} Y^{k}: k=0, \ldots, n\right\}\right)$. The pioneers of invariant theory also studied "covariants", which are polynomials in both the coefficients $a_{i}$ and the variables $X$ and $Y$ themselves which are fixed under the action of $G$. In modern notation, the algebra of covariants is $\mathbb{k}\left[U_{n} \oplus U_{1}^{*}\right]^{G}$. There is, in fact, an even stronger connection between covariants and the basic actions of $\mathbb{G}_{a}$ : the algebras $\mathbb{k}\left[U_{n} \oplus U_{1}^{*}\right]^{G}$ and $\mathbb{k}\left[U_{n}\right]^{\mathbb{G}_{a}}$ are actually isomorphic. Let us identify the algebra $\mathbb{k}\left[U_{n} \oplus U_{1}^{*}\right]$ with the polynomial ring $\mathbb{k}\left[a_{0}, a_{1}, \ldots, a_{n}, X, Y\right]$ (we abuse notation by using the same letters $a_{i}$ for coordinates and coordinate functions). Define a mapping $\Phi: \mathbb{k}\left[U_{n} \oplus U_{1}^{*}\right]^{G} \rightarrow \mathbb{k}\left[U_{n}\right]^{\mathbb{G}_{a}}$ by

$$
\Phi\left(f\left(a_{0}, a_{1}, a_{2}, \ldots, a_{n}, X, Y\right)\right):=f\left(a_{0}, a_{1}, a_{2}, \ldots, a_{n}, 0,1\right) .
$$

The theorem of Roberts 19 states that $\Phi$ is an isomorphism. In classical invariant theory one often studies the basic actions of $\mathbb{G}_{a}$ in order to get a handle on the covariants of binary forms using Roberts' isomorphism. One word of caution is needed at this point. While [16, Proposition 1] implies that a separating set for $\mathbb{k}\left[U_{n} \oplus U_{1}^{*}\right]^{\mathrm{SL}_{2}(\mathbb{k})}$ must be mapped under $\Phi$ to a separating set for $\mathbb{k}\left[U_{n}\right]^{\mathbb{G}_{a}}$, the converse is not necessarily true, so the separating sets we construct in this paper most likely do not lift to give separating sets for the covariants of binary forms (cf. [16, Remark 3]).

However, we owe the following observation to the anonymous referee: Roberts' isomorphism induces a bijection between the $\mathrm{SL}_{2}(\mathbb{k})$ orbits in $U_{n} \times\left(U_{1}^{*} \backslash\{0\}\right)$ and the $\mathbb{G}_{a}$ orbits in $U_{n}$. In fact, for $(u,(x, y)) \in U_{n} \times\left(U_{1}^{*} \backslash\{0\}\right)$ choose $\sigma \in \mathrm{SL}_{2}(\mathbb{k})$ with $\sigma(x, y)=(0,1)$. Then the orbit $\mathrm{SL}_{2}(\mathbb{k}) \cdot(u,(x, y))$ corresponds to $\mathbb{G}_{a} \cdot \sigma(u)$. Now, 
if for a separating set $S \subseteq \mathbb{k}\left[U_{n}\right]^{\mathbb{G}_{a}}$ and $v, w \in U_{n} \times\left(U_{1}^{*} \backslash\{0\}\right)$ we have $f(v)=f(w)$ for all $f \in \Phi^{-1}(S)$, then $f(v)=f(w)$ for all $f \in \mathbb{k}\left[U_{n} \oplus U_{1}^{*}\right]^{\mathrm{SL}_{2}(\mathbb{k})}$. In other words, $\Phi^{-1}(S)$ separates outside $U_{n} \times\{0\} \subseteq U_{n} \oplus U_{1}^{*}$.

We now state our main results. For any real number $x$, the symbol $[x]$ denotes the largest integer less than or equal to $x$. We begin by definining some important invariants, namely

$$
f_{m}:=\sum_{k=0}^{m-1}(-1)^{k} x_{k} x_{2 m-k}+\frac{1}{2}(-1)^{m} x_{m}^{2} \in \operatorname{ker} D_{n} \quad \text { for } m=1, \ldots,\left[\frac{n}{2}\right]
$$

and $f_{0}:=x_{0}$. Note that these elements are semitransvectants; see Section 5 Further, we define the elements

$$
s_{m}:=\sum_{k=0}^{m}(-1)^{k} \frac{2 m+1-2 k}{2} x_{k} x_{2 m+1-k} \in R_{n} \quad \text { for } m=1, \ldots,\left[\frac{n-1}{2}\right]
$$

and $s_{0}:=x_{1}$, which satisfy

$$
D_{n} s_{m}=f_{m} \quad \text { for } m=0, \ldots,\left[\frac{n-1}{2}\right],
$$

and in particular $D_{n} s_{m} \in A_{n} \backslash\{0\}$. Elements with this property are called local slices.

For any $a \in R_{n} \backslash\{0\}$, let $\nu(a)$ denote the nilpotency index $\nu(a):=\min \{m \in \mathbb{N}$ : $\left.D_{n}^{m+1}(a)=0\right\}$, and $\nu(0):=-\infty$. If $s \in R_{n}$ is a local slice, then for any $a \in R_{n}$ we define

$$
\begin{aligned}
\epsilon_{s}(a) & :=\left.\left(\exp \left(t D_{n}\right) a\right)\right|_{t:=-s / D_{n} s} \cdot\left(D_{n} s\right)^{\nu(a)} \\
& =\sum_{k=0}^{\nu(a)} \frac{(-1)^{k}}{k !}\left(D_{n}^{k} a\right) s^{k}\left(D_{n} s\right)^{\nu(a)-k} \in A_{n} .
\end{aligned}
$$

By the Slice Theorem [12, Corollary 1.22], we have

$$
A_{n} \subseteq \mathbb{k}\left[\epsilon_{s}\left(x_{0}\right), \ldots, \epsilon_{s}\left(x_{n}\right)\right]_{D_{n} s} .
$$

When $s=x_{1}$ and so $D_{n} s=x_{0}$, this is the first stage in Lin Tan's (and van den Essen's) algorithm for producing a generating set for $A_{n}[22,23$.

Theorem 2.1. Given $n$, we define a set $E_{n}$ consisting of the following elements:

$$
\begin{gathered}
f_{0}, f_{1}, \ldots, f_{\left[\frac{n}{2}\right]}, \\
\epsilon_{s_{0}}\left(x_{2}\right), \ldots, \epsilon_{s_{0}}\left(x_{n}\right), \\
\epsilon_{s_{1}}\left(x_{1}\right), \ldots, \epsilon_{s_{1}}\left(x_{n}\right), \\
\epsilon_{s_{2}}\left(x_{2}\right), \ldots, \epsilon_{s_{2}}\left(x_{n}\right), \\
\epsilon_{s_{3}}\left(x_{3}\right), \ldots, \epsilon_{s_{3}}\left(x_{n}\right), \\
\vdots \\
\epsilon_{s_{\left[\frac{n-1}{2}\right]}}\left(x_{\left[\frac{n-1}{2}\right]}\right), \ldots, \epsilon_{s_{\left[\frac{n-1}{2}\right]}}\left(x_{n}\right) .
\end{gathered}
$$

If $n \equiv 0 \bmod 4$ we also append to $E_{n}$ an extra invariant $w$ which is defined in Lemma 5.4. Then the set $E_{n}$ is a separating set for $A_{n}$. 
Note that $\epsilon_{s_{0}}\left(x_{0}\right)=f_{0}$ and $\epsilon_{s_{0}}\left(x_{1}\right)=0$. The size of this separating set is about $\frac{3}{8} n^{2}$. The following table shows its exact size for some values of $n$. The lower line gives the size $c_{n}$ of a minimal generating set for $A_{n}$; see Olver [17, p. 40]. Olver says that this list cannot be trusted for $n \geq 7$. For $c_{7}$, we use Bedratyuk's value $c_{7}=147$ [2], while in Olver's list, values $c_{7}=124$ or $c_{7}=130$ are offered, depending on the source. We also want to remark that for $n \geq 5$, we could save 5 elements by replacing the 10 elements of $E_{4} \backslash\{w\}$ appearing in $E_{n}$ by the 5 generators of $A_{4}$. (For $n=4$ we could save 6 elements.) Note that generators for $n \leq 7$ are listed explicitly in [3, 2].

\begin{tabular}{c|c|c|c|c|c|c|c|c|c|c|c|c|c|c|c|c|c}
$n$ & 4 & 5 & 6 & 7 & 8 & 9 & 10 & 11 & 12 & 13 & 14 & 15 & 16 & 17 & 18 & 19 & 20 \\
\hline$\left|E_{n}\right|$ & 11 & 16 & 20 & 28 & 34 & 43 & 49 & 61 & 69 & 82 & 90 & 106 & 116 & 133 & 143 & 163 & 175 \\
\hline$c_{n}$ & 5 & 23 & 26 & 147 & 69 & 415 & 475 & 949 & $?$ & $?$ & $?$ & $?$ & $?$ & $?$ & $?$ & $?$ & $?$
\end{tabular}

It is also worth noting that our separating set consists of invariants whose degree is at most $2 n+1$.

\section{The Radical of the Hilbert ideal}

Let $R_{n}, D_{n}$ and $A_{n}$ be as in the introduction. For any $m<n$ we have the algebra homomorphism

$$
\pi_{m, n}: R_{n} \rightarrow R_{m}, \quad f\left(x_{0}, x_{1}, \ldots, x_{n}\right) \mapsto f(\underbrace{0, \ldots, 0}_{n-m \text { times }}, x_{0}, \ldots, x_{m}),
$$

which satisfies $\pi_{m, n} \circ D_{n}=D_{m} \circ \pi_{m, n}$ and thus induces a map $A_{n} \rightarrow A_{m}$.

Consider the Hilbert ideal $I_{n}:=A_{n,+} R_{n} \unlhd R_{n}$. With the invariants $f_{m}$ defined in (2), we get the following inclusion for its radical:

$$
\left(x_{0}, \ldots, x_{\left[\frac{n}{2}\right]}\right) R_{n}=\sqrt{\left(f_{0}, f_{1}, \ldots, f_{\left[\frac{n}{2}\right]}\right) R_{n}} \subseteq \sqrt{I_{n}} .
$$

The main purpose of this section is to prove that the reverse inclusion holds too.

\section{Proposition 3.1. $\quad$ (a) The radical of the Hilbert ideal is given by}

$$
\sqrt{I_{n}}=\left(x_{0}, \ldots, x_{\left[\frac{n}{2}\right]}\right) R_{n} .
$$

(b) $\pi_{n-\left[\frac{n}{2}\right]-1, n}\left(A_{n}\right)=\mathbb{k}$.

(c) $\pi_{m, 2 m}\left(A_{2 m}\right)=\mathbb{k}\left[x_{0}^{2}\right]$ for $m$ odd.

(d) $\pi_{m, 2 m}\left(A_{2 m}\right)=\mathbb{k}\left[x_{0}^{2}, x_{0}^{3}\right]$ for $m$ even.

Proof. We will make use of Roberts' isomorphism as defined in the previous section, with the only difference that we will choose variables so that the $\mathbb{G}_{a}$-actions become basic, using the notation of the introduction. Additionally, let $\mathbb{G}_{a}$ act basically on $\left\langle y_{0}, y_{1}\right\rangle_{\mathbb{k}}$. The action of $\mathbb{G}_{a}$ on $\tilde{R}_{n}:=R_{n}\left[y_{0}, y_{1}\right]$ extends to an action of $\mathrm{SL}_{2}(\mathbb{k})$ on $\tilde{R}_{n}$ such that the following holds:

(1) for any $a \in \mathbb{K} \backslash\{0\}$ and $\mu_{a}:=\left(\begin{array}{cc}a^{-1} & 0 \\ 0 & a\end{array}\right) \in \mathrm{SL}_{2}$ we have $\mu_{a}\left(x_{k}\right)=a^{2 k-n} x_{k}$ for $k=0, \ldots, n$ and $\mu_{a}\left(y_{k}\right)=a^{2 k-1} y_{k}$ for $k=0,1$;

(2) for $\tau:=\left(\begin{array}{cc}0 & -1 \\ 1 & 0\end{array}\right) \in \mathrm{SL}_{2}$ we have $\tau\left(x_{k}\right)=(-1)^{k} \frac{(n-k) !}{k !} x_{n-k}$ for $k=0, \ldots, n$ and $\tau\left(y_{k}\right)=(-1)^{k} y_{1-k}$ for $k=0,1$.

Recall from section 2 that the algebra map

$$
\Phi: \tilde{R}_{n} \rightarrow R_{n}, \quad f\left(x_{0}, \ldots, x_{n}, y_{0}, y_{1}\right) \mapsto f\left(x_{0}, \ldots, x_{n}, 0,1\right)
$$


induces an isomorphism of invariant rings $\tilde{R}_{n}^{\mathrm{SL}_{2}} \rightarrow R_{n}^{\mathbb{G}_{a}}=A_{n}$. Now let $f \in A_{n}$ and $F \in \tilde{R}_{n}^{\mathrm{SL}_{2}}$ with $\Phi(F)=f$. Then we also have $f=\Phi\left(\mu_{a} \cdot F\right)$ for all $a \in \mathbb{k} \backslash\{0\}$, i.e.

$$
f\left(x_{0}, \ldots, x_{n}\right)=F\left(a^{-n} x_{0}, a^{-n+2} x_{1}, \ldots, a^{n} x_{n}, 0, a\right) \text { for all } a \in \mathbb{k} \backslash\{0\} .
$$

Thus,

$\pi_{n-\left[\frac{n}{2}\right]-1, n}(f)=F\left(0, \ldots, 0, a^{2\left(\left[\frac{n}{2}\right]+1\right)-n} x_{0}, \ldots, a^{n} x_{n-\left[\frac{n}{2}\right]-1}, 0, a\right)$ for all $a \in \mathbb{k} \backslash\{0\}$,

and since this equation is polynomial in $a$ and $\mathbb{k}$ is an infinite field, it also holds for $a=0$. Therefore, $\pi_{n-\left[\frac{n}{2}\right]-1, n}(f)=F(0, \ldots, 0) \in \mathbb{k}$, which proves (a) and (b). Similarly, for $n=2 m$ we find

$$
\pi_{m, 2 m}(f)=F\left(0, \ldots, 0, x_{0}, a^{2} x_{1}, \ldots, a^{2 m} x_{m}, 0, a\right) \text { for all } a \in \mathbb{k} \backslash\{0\},
$$

which is again a polynomial in $a$. When $a=0$, we get

$$
\pi_{m, 2 m}(f)=F\left(0, \ldots, 0, x_{0}, 0, \ldots, 0\right)=p\left(x_{0}\right)
$$

for some polynomial $p\left(x_{0}\right) \in \mathbb{k}\left[x_{0}\right]$, so $\pi_{m, 2 m}\left(A_{2 m}\right) \subseteq \mathbb{k}\left[x_{0}\right]$. Since $\pi_{m, 2 m}\left(f_{m}\right)=$ $\frac{1}{2}(-1)^{m} x_{0}^{2}$, we get the inclusion $\mathbb{k}\left[x_{0}^{2}\right] \subseteq \pi_{m, 2 m}\left(A_{2 m}\right)$. Using that $F$ is also invariant under $\tau$, we find in the same manner as before that

$$
\pi_{m, 2 m}(f)=\left.\pi_{m, 2 m}\left(\Phi\left(\tau \mu_{a^{-1}} F\right)\right)\right|_{a=0}=F\left(0, \ldots, 0,(-1)^{m} x_{0}, 0, \ldots, 0\right)=p\left((-1)^{m} x_{0}\right) .
$$

Therefore, for $m$ odd we get $\pi_{m, 2 m}(f)=p\left(x_{0}\right)=p\left(-x_{0}\right) \in \mathbb{k}\left[x_{0}^{2}\right]$, which proves (c). For $m$ even, to prove (d), we refer to Lemma 5.4, which gives a $w \in A_{2 m}$ with $\pi_{m, 2 m}(w)=x_{0}^{3}$, so $\mathbb{k}\left[x_{0}^{2}, x_{0}^{3}\right] \subseteq \pi_{m, 2 m}\left(A_{2 m}\right) \subseteq \mathbb{k}\left[x_{0}\right]$. Since $x_{0}$ generates the degree one elements of $A_{2 m}$ and $\pi_{m, 2 m}\left(x_{0}\right)=0$, we are done.

We want to mention here that the method of proof for Proposition [3.1 (a) also works for decomposable actions. Consider

$$
R:=\mathbb{k}\left[x_{0,1}, \ldots, x_{n_{1}, 1}, \ldots, x_{0, k}, \ldots, x_{n_{k}, k}\right]
$$

and $D=D_{n_{1}}+\cdots+D_{n_{k}}$ with $D_{n_{i}}=x_{0, i} \frac{\partial}{\partial x_{1, i}}+\cdots+x_{n_{i}-1, i} \frac{\partial}{\partial x_{n_{i}, i}}$. Using an algebra homomorphism $\pi$ which behaves on each subalgebra $\mathbb{k}\left[x_{0, i}, \ldots, x_{n_{i}, i}\right]$ as $\pi_{n_{i}-\left[\frac{n_{i}}{2}\right]-1, n_{i}}$, we get with the same proof

Theorem 3.2. The radical of the Hilbert ideal of ker $D$ is given by

$$
\left(x_{0,1}, \ldots, x_{\left[\frac{n_{1}}{2}\right], 1}, \ldots, x_{0, k}, \ldots, x_{\left[\frac{n_{k}}{2}\right], k}\right) R .
$$

\section{Construction of a separating Set}

In this section, we prove our main result.

Proof of Theorem 2.1 . For $V_{n}=\mathbb{k}^{n+1}$ with $\mathbb{k}\left[V_{n}\right]=R_{n}$, assume there are two elements $a=\left(a_{0}, \ldots, a_{n}\right)$ and $b=\left(b_{0}, \ldots, b_{n}\right)$ of $V_{n}$ such that $f(a)=f(b)$ for all $f \in E_{n}$. We have to show that $f(a)=f(b)$ for all $f \in A_{n}$. As $x_{0} \in E_{n}$, we have $a_{0}=b_{0}$. Assume first $a_{0}=b_{0} \neq 0$. By the Slice Theorem, $A_{n} \subseteq \mathbb{k}\left[E_{n}\right]_{x_{0}}$. Therefore, $f \in A_{n}$ can be written as $f=\frac{p}{x_{0}^{l}}$ with $p \in \mathbb{k}\left[E_{n}\right]$ and $l \geq 0$. By assumption, $p(a)=p(b)$ and $a_{0}^{l}=b_{0}^{l} \neq 0$, so $f(a)=p(a) / a_{0}^{l}=p(b) / b_{0}^{l}=f(b)$. Now assume $a_{0}=b_{0}=0$ and let $m$ be maximal such that $a_{0}=a_{1}=\cdots=a_{m}=0$, so $a_{m+1} \neq 0$ (if $m<n$ ). By induction on $k$, we shall show that $b_{k}=0$ for 
$k=0, \ldots, \min \left\{m,\left[\frac{n}{2}\right]\right\}$. By assumption this holds for $k=0$, so assume it holds for some $k<\min \left\{m,\left[\frac{n}{2}\right]\right\}$. Then

$$
\frac{(-1)^{k+1}}{2} a_{k+1}^{2}=f_{k+1}(a)=f_{k+1}(b)=\frac{(-1)^{k+1}}{2} b_{k+1}^{2},
$$

so $b_{k+1}=0$ since $a_{k+1}=0$. If $m \geq\left[\frac{n}{2}\right]$, then $f(a)=f(0)=f(b)$ for any $f \in A_{n}$ by Proposition [3.1, so now assume $0 \leq m<\left[\frac{n}{2}\right]$. Equation (5) for $k=m$ shows that $0 \neq a_{m+1}^{2}=b_{m+1}^{2}$. We now distinguish different cases.

Case 1: $m<\left[\frac{n-1}{2}\right]$. Then $s_{m+1}$ is defined, and by the Slice Theorem,

$$
A_{n} \subseteq \mathbb{k}\left[\epsilon_{s_{m+1}}\left(x_{0}\right), \ldots, \epsilon_{s_{m+1}}\left(x_{n}\right)\right]_{f_{m+1}} .
$$

Applying $\pi:=\pi_{n-m-1, n}$ on both sides yields

$$
\pi\left(A_{n}\right) \subseteq \mathbb{k}\left[\pi\left(\epsilon_{s_{m+1}}\left(x_{m+1}\right)\right), \ldots, \pi\left(\epsilon_{s_{m+1}}\left(x_{n}\right)\right)\right]_{\pi\left(f_{m+1}\right)},
$$

where we used that $\pi_{n-m-1, n}\left(\epsilon_{s_{m+1}}\left(x_{k}\right)\right)=0$ for $k=0, \ldots, m$. The right-hand side is included in $\mathbb{k}\left[\pi\left(E_{n}\right)\right]_{\pi\left(f_{m+1}\right)}$. Therefore, for any $f \in A_{n}$ there is $p \in \mathbb{k}\left[E_{n}\right]$ and $l \geq 0$ such that $\pi(f)=\frac{\pi(p)}{\pi\left(f_{m+1}\right)^{l}}$. Let

$$
\gamma: V_{n} \rightarrow V_{n-m-1}, \quad\left(c_{0}, \ldots, c_{n}\right) \mapsto\left(c_{m+1}, \ldots, c_{n}\right) .
$$

Then

$$
\begin{aligned}
f(a) & =\pi(f)(\gamma(a))=\frac{\pi(p)}{\pi\left(f_{m+1}\right)^{l}}(\gamma(a))=\frac{\pi(p)(\gamma(a))}{\left(\pi\left(f_{m+1}\right)(\gamma(a))\right)^{l}}=\frac{p(a)}{f_{m+1}(a)^{l}} \\
& =\frac{p(b)}{f_{m+1}(b)^{l}}=\frac{\pi(p)(\gamma(b))}{\left(\pi\left(f_{m+1}\right)(\gamma(b))\right)^{l}}=\pi(f)(\gamma(b))=f(b) .
\end{aligned}
$$

Here we used that the elements $p$ and $f_{m+1}$ of $E_{n}$ take the same value on $a, b$ by assumption, and $f_{m+1}(a)=f_{m+1}(b) \neq 0$.

Case 2: $\left[\frac{n-1}{2}\right]=m<\left[\frac{n}{2}\right]$. In this case, $n$ has to be even, and $n=2 m^{\prime}$ with $m^{\prime}=m+1$. Let $\pi$ and $\gamma$ be as before, so $\pi=\pi_{m^{\prime}, 2 m^{\prime}}$ and $\gamma: V_{2 m^{\prime}} \rightarrow V_{m^{\prime}}$. First assume $m^{\prime}$ is odd. By Proposition 3.1 (c) we have

$$
\pi\left(A_{n}\right)=\mathbb{k}\left[x_{0}^{2}\right]=\mathbb{k}\left[\pi\left(f_{m^{\prime}}\right)\right] .
$$

If $m^{\prime}$ is even, by Proposition 3.1 (d) we have

$$
\pi\left(A_{n}\right)=\mathbb{k}\left[x_{0}^{2}, x_{0}^{3}\right]=\mathbb{k}\left[\pi\left(f_{m^{\prime}}\right), \pi(w)\right],
$$

with $w$ the element of Lemma 5.4. In both cases, $\pi\left(A_{n}\right)=\mathbb{k}\left[\pi\left(E_{n}\right)\right]$, so for any $f \in A_{n}$, there exists $p \in \mathbb{k}\left[E_{n}\right]$ such that $\pi(f)=\pi(p)$. Therefore,

$$
f(a)=\pi(f)(\gamma(a))=\pi(p)(\gamma(a))=p(a)=p(b)=\pi(p)(\gamma(b))=\pi(f)(\gamma(b))=f(b) .
$$

We have shown: for any $f \in A_{n}$ we have $f(a)=f(b)$, and so we are done.

\section{The existence of $w$}

In this section we prove Lemma 5.4, which requires some more machinery. Note that we need this lemma in order to construct a separating set only in the case where $n \equiv 0 \bmod 4$; in the other cases, $w$ is not contained in our separating set. We will make use of semitransvectants, which are the classical transvectants transformed under Roberts' isomorphism; see for example 2, 4, 17. Recall that for a covariant $F \in \tilde{R}_{n}^{\mathrm{SL}_{2}}=R_{n}\left[y_{0}, y_{1}\right]^{\mathrm{SL}_{2}}$, its total degree in $y_{0}, y_{1}$ is called the order of $F$. For 
covariants $F$ and $G$ of orders $l$ and $m$ respectively, we can construct new covariants given by

$$
\langle F, G\rangle^{(r)}:=\sum_{k=0}^{r}(-1)^{k}\left(\begin{array}{l}
r \\
k
\end{array}\right) \frac{\partial^{r} F}{\partial y_{0}^{r-k} \partial y_{1}^{k}} \frac{\partial^{r} G}{\partial y_{0}^{k} \partial y_{1}^{r-k}}, \quad r \leq \min (l, m),
$$

which is called the $r$ th transvectant of $F$ and $G$ (see [17, p. 88]). Transvectants play a key role in Gordan's famous proof of the finite generation of covariants of binary forms 13. The transformation of this construction under Roberts' isomorphism leads the following definition (see also [1]).

Definition 5.1. Let $\Phi: R_{n}\left[y_{0}, y_{1}\right]^{\mathrm{SL}_{2}} \rightarrow R_{n}^{\mathbb{G}_{a}}=A_{n}$ be Roberts' isomorphism, given by substituting $y_{0}:=0, y_{1}:=1$. Let $f$ and $g$ be a pair of invariants in $A_{n}$. Then for $r \leq \min (l, m)$, where $l$ and $m$ are the orders of $\Phi^{-1}(f)$ and $\Phi^{-1}(g)$ as above, we define the $r$ th semitransvectant of $f$ and $g$ by

$$
[f, g]^{(r)}:=\Phi\left(\left\langle\Phi^{-1}(f), \Phi^{-1}(g)\right\rangle^{(r)}\right) .
$$

In order to get an explicit expression for the semitransvectant, we introduce a second derivation on $R_{n}$, which is somewhat inverse to $D_{n}$ :

$$
\Delta_{n}:=\sum_{k=0}^{n}(n-k)(k+1) x_{k+1} \frac{\partial}{\partial x_{k}} .
$$

This derivation comes from the other canonical embedding of $\mathbb{G}_{a}$ in $\mathrm{SL}_{2}$; namely for $f \in R_{n}, a \in \mathbb{k}$ we have $\left(\begin{array}{cc}1 & \\ a & 1\end{array}\right) * f=\exp \left(a \Delta_{n}\right) f$. Let $\operatorname{ord}(f)$ denote the nilpotency index of $f$ with respect to $\Delta_{n}$. Assume $F \in R_{n}\left[y_{0}, y_{1}\right]^{\mathrm{SL}_{2}}$ is homogeneous of degree $m$ in the variables $y_{0}, y_{1}$, so it can be written in the form $F=f y_{1}^{m}+y_{0} \cdot(\ldots)$ with $f \in R_{n}$. Then $\Phi(F)=f$, and invariance of $F$ under the torus action implies that all terms $x_{0}^{a_{0}} \ldots x_{n}^{a_{n}}$ in $f$ satisfy $m=\sum_{k=0}^{n}(n-2 k) a_{k}$. A polynomial $f \in R_{n}^{\mathbb{G}_{a}}$ with this property is called isobaric of weight $m$, and then we have $m=\operatorname{ord}(f)$. For an isobaric $f \in R_{n}^{\mathbb{G}_{a}}$, by [14, p. 43] the inverse of Roberts' isomorphism is given by

$$
\Phi^{-1}(f)=\sum_{i=0}^{\operatorname{ord}(f)}(-1)^{i} \frac{\Delta_{n}^{i}(f)}{i !} y_{0}^{i} y_{1}^{\operatorname{ord}(f)-i}
$$

Proposition 5.2. Let $f, g \in R_{n}^{\mathbb{G}_{a}}$ be isobaric. Then for $r \leq \min (\operatorname{ord}(f)$, ord $(g))$, the rth semitransvectant of $f$ and $g$ is given by the formula

$$
[f, g]^{(r)}=\sum_{k=0}^{r}(-1)^{k}\left(\begin{array}{c}
r \\
k
\end{array}\right) \Delta_{n}^{k}(f) \frac{(\operatorname{ord}(f)-k) !}{(\operatorname{ord}(f)-r) !} \Delta_{n}^{r-k}(g) \frac{(\operatorname{ord}(g)-r+k) !}{(\operatorname{ord}(g)-r) !}
$$

Proof. Let $\frac{\Delta_{n}^{i}(f)}{i !}:=\lambda_{i}$ and $\frac{\Delta_{n}^{i}(g)}{i !}:=\mu_{i}$. Then

$$
\frac{\partial^{r} \Phi^{-1}(f)}{\partial y_{0}^{r-k} \partial y_{1}^{k}}=\sum_{i=r-k}^{\operatorname{ord}(f)-k}(-1)^{i} \lambda_{i} \frac{i !}{(i-r+k) !} \frac{(\operatorname{ord}(f)-i) !}{(\operatorname{ord}(f)-i-k) !} y_{0}^{i-r+k} y_{1}^{\operatorname{ord}(f)-i-k}
$$

and

$$
\frac{\partial^{r} \Phi^{-1}(g)}{\partial y_{0}^{k} \partial y_{1}^{r-k}}=\sum_{i=k}^{\operatorname{ord}(g)-r+k}(-1)^{i} \mu_{i} \frac{i !}{(i-k) !} \frac{(\operatorname{ord}(g)-i) !}{(\operatorname{ord}(g)-i-r+k) !} y_{0}^{i-k} y_{1}^{\operatorname{ord}(g)-i-r+k}
$$


therefore

$$
\Phi\left(\frac{\partial^{r} \Phi^{-1}(f)}{\partial y_{0}^{r-k} \partial y_{1}^{k}}\right)=(-1)^{r-k} \lambda_{r-k} \frac{(r-k) !(\operatorname{ord}(f)-r+k) !}{(\operatorname{ord}(f)-r) !}
$$

and

$$
\Phi\left(\frac{\partial^{r} \Phi^{-1}(g)}{\partial y_{0}^{k} \partial y_{1}^{r-k}}\right)=(-1)^{k} \mu_{k} \frac{k !(\operatorname{ord}(g)-k) !}{(\operatorname{ord}(g)-r) !} .
$$

Using the fact that $\Phi$ is an algebra homomorphism we have

$$
\begin{aligned}
{[f, g]^{(r)} } & =\sum_{k=0}^{r}(-1)^{k+r}\left(\begin{array}{c}
r \\
k
\end{array}\right) \lambda_{r-k} \frac{(r-k) !(\operatorname{ord}(f)-r+k) !}{(\operatorname{ord}(f)-r) !} \mu_{k} \frac{k !(\operatorname{ord}(g)-k) !}{(\operatorname{ord}(g)-r) !} \\
& =\sum_{k=0}^{r}(-1)^{k+r}\left(\begin{array}{c}
r \\
k
\end{array}\right) \Delta_{n}^{r-k}(f) \frac{(\operatorname{ord}(f)-r+k) !}{(\operatorname{ord}(f)-r) !} \Delta_{n}^{k}(g) \frac{(\operatorname{ord}(g)-k) !}{(\operatorname{ord}(g)-r) !} \\
& =\sum_{k=0}^{r}(-1)^{k}\left(\begin{array}{c}
r \\
k
\end{array}\right) \Delta_{n}^{k}(f) \frac{(\operatorname{ord}(f)-k) !}{(\operatorname{ord}(f)-r) !} \Delta_{n}^{r-k}(g) \frac{(\operatorname{ord}(g)-r+k) !}{(\operatorname{ord}(g)-r) !}
\end{aligned}
$$

as required.

Remark 5.3. This is analogous to [1, Lemma 1], using a different basis.

The formula shows that, up to some scalar factor, $f_{m}$ (from (21)) equals $\left[x_{0}, x_{0}\right]^{(2 m)}$ (while $\left[x_{0}, x_{0}\right]^{(r)}=0$ for $r$ odd), and $\epsilon_{s_{m}}\left(x_{1}\right)$ equals $\left[x_{0}, f_{m}\right]^{(1)}$. We wonder whether there is also a connection between $\epsilon_{s_{m}}\left(x_{j}\right)$ and $\left[x_{0}, f_{m}^{j}\right]^{(j)}$.

Lemma 5.4. Suppose $n$ is divisible by 4 , so $n=2 m=4 p$. Then there is an invariant $w \in A_{n}$ satisfying $\pi_{m, n}(w)=x_{0}^{3}$.

Proof. Throughout the proof we use the shorthand $\pi:=\pi_{m, n}$, and we set $f:=f_{p}=$ $\frac{1}{2} \sum_{i=0}^{m}(-1)^{i} x_{i} x_{m-i}$ (which is proportional to $\left[x_{0}, x_{0}\right]^{(m)}$ ). Obviously, $f$ is isobaric of weight $(n-2 i)+(n-2(m-i))=2 n-2 m=n=\operatorname{ord}(f)$. Thus we may define

$$
\bar{w}:=\left[x_{0}, f\right]^{(n)}=\sum_{k=0}^{n}(-1)^{k} \frac{n !^{2} k !}{(n-k) !} x_{k} \Delta_{n}^{n-k}(f)=\sum_{k=0}^{n}(-1)^{k} \frac{n !^{2}(n-k) !}{k !} x_{n-k} \Delta_{n}^{k}(f),
$$

where we used Proposition 5.2, Thus,

$$
\pi(\bar{w})=\sum_{k=0}^{m}(-1)^{k} \frac{n !^{2}(n-k) !}{k !} x_{m-k} \pi\left(\Delta_{n}^{k}(f)\right) .
$$

Using Leibniz's formula for iterated differentiation of products, we have

$$
\begin{gathered}
\pi\left(\Delta_{n}^{k}\left(x_{i} x_{m-i}\right)\right)=\sum_{j=0}^{k}\left(\begin{array}{c}
k \\
j
\end{array}\right) \pi\left(\Delta_{n}^{j} x_{i}\right) \pi\left(\Delta_{n}^{k-j} x_{m-i}\right) \\
=\sum_{j=m-i}^{k-i}\left(\begin{array}{c}
k \\
j
\end{array}\right) \frac{(i+j) !(n-i) !}{i !(n-i-j) !} \pi\left(x_{i+j}\right) \frac{(m-i+k-j) !(m+i) !}{(m-i) !(m+i-k+j) !} \pi\left(x_{m-i+k-j}\right) \\
=\sum_{j=0}^{k-m}\left(\begin{array}{c}
k \\
j+m-i
\end{array}\right) \frac{(m+j) !(n-i) !}{i !(m-j) !} \pi\left(x_{m+j}\right) \frac{(k-j) !(m+i) !}{(m-i) !(n-k+j) !} \pi\left(x_{k-j}\right) .
\end{gathered}
$$


In particular, $\pi\left(\Delta_{n}^{k}\left(x_{i} x_{m-i}\right)\right)=0$ for all $k<m$, and since $f$ is a linear combination of terms of the form $x_{i} x_{m-i}$, we have $\pi\left(\Delta_{n}^{k}(f)\right)=0$ for all $k<m$. From this, remembering $m$ is even, it follows that $\pi(\bar{w})=n !^{2} x_{0} \pi\left(\Delta_{n}^{m}(f)\right)$. Therefore, since

$$
\pi\left(\Delta_{n}^{m}\left(x_{i} x_{m-i}\right)\right)=\left(\begin{array}{c}
m \\
m-i
\end{array}\right) x_{0}^{2} \frac{(n-i) !}{i !} \frac{(m+i) !}{(m-i) !}
$$

and $f=\frac{1}{2} \sum_{i=0}^{m}(-1)^{i} x_{i} x_{m-i}$, we obtain

$$
\begin{aligned}
\pi\left(\Delta_{n}^{m}(f)\right) & =\frac{1}{2} x_{0}^{2} \sum_{i=0}^{m}(-1)^{i}\left(\begin{array}{c}
m \\
i
\end{array}\right) \frac{(n-i) !}{i !} \frac{(m+i) !}{(m-i) !} \\
& =\frac{((2 p) !)^{2}}{2} x_{0}^{2} \sum_{i=0}^{2 p}(-1)^{i}\left(\begin{array}{c}
2 p \\
i
\end{array}\right)\left(\begin{array}{c}
4 p-i \\
2 p
\end{array}\right)\left(\begin{array}{c}
2 p+i \\
i
\end{array}\right) .
\end{aligned}
$$

Thus, $\pi(\bar{w})=n !^{2} x_{0} \pi\left(\Delta_{n}^{m}(f)\right)$ is a nonzero multiple of $x_{0}^{3}$ if the sum above is nonzero. This follows from Lemma 5.6.

Remark 5.5. With $g:=\Delta_{n}^{n}(f)$, we have $\bar{w}=c \cdot \sum_{k=0}^{n}(-1)^{k} x_{k} D^{k} g$ with $c \in \mathbb{k}$.

Lemma 5.6. For all $p \geq 1$ we have

$$
\sum_{k=0}^{2 p}(-1)^{k}\left(\begin{array}{c}
2 p \\
k
\end{array}\right)\left(\begin{array}{c}
4 p-k \\
2 p
\end{array}\right)\left(\begin{array}{c}
2 p+k \\
k
\end{array}\right)=(-1)^{p} \frac{(3 p) !}{(p !)^{3}} .
$$

Proof. The argument which follows was produced using the implementation of Zeilberger's algorithm [25] in the remarkable EKHAD package for Maple [18]. Let

$$
F(p, k):=\left\{(-1)^{k}\left(\begin{array}{c}
2 p \\
k
\end{array}\right)\left(\begin{array}{c}
4 p-k \\
2 p \\
0
\end{array}\right)\left(\begin{array}{c}
2 p+k \\
k
\end{array}\right) \begin{array}{c}
0 \leq k \leq 2 p, \\
\text { otherwise }
\end{array}\right.
$$

and let $S(p):=\sum_{k=0}^{2 p} F(p, k)$. We show that the following recurrence relation holds:

$$
6(3 p+2)(3 p+1) S(p)+2(p+1)^{2} S(p+1)=0 .
$$

To do this, we consider the function

$$
\begin{aligned}
& G(p, k):=\frac{1}{2} k^{2}\left(180-184 k+1036 p+59 k^{2}+2192 p^{2}-790 p k-1116 p^{2} k+168 p k^{2}\right. \\
& \left.+2024 p^{3}-8 k^{3}+688 p^{4}+k^{4}-520 p^{3} k+120 p^{2} k^{2}-10 p k^{3}\right)(-4 p+k-1) \frac{F(p, k)}{R(p, k)},
\end{aligned}
$$

where $R(p, k)=(2 p+1)(-2 p-2+k)^{2}(-2 p-1+k)^{2}$. For $0 \leq k \leq 2 p-1$ it satisfies the relation

$$
G(p, k+1)-G(p, k)=6(3 p+2)(3 p+1) F(p, k)+2(p+1)^{2} F(p+1, k) .
$$

Summing both sides over $0 \leq k \leq 2 p-1$ (and adding remaining terms) produces (6)), and an easy inductive argument then shows that $S(p)=(-1)^{p} \frac{(3 p) !}{(p !)^{3}}$.

Remark 5.7. The sum $S(p)$ is the well-poised hypergeometric series

$$
\left(\begin{array}{c}
4 p \\
2 p
\end{array}\right) \sum_{k=0}^{\infty} \frac{(-2 p)_{k}(2 p+1)_{k}(-2 p)_{k}}{(1)_{k}(-4 p)_{k} k !}=\left(\begin{array}{c}
4 p \\
2 p
\end{array}\right){ }_{3} F_{2}[-2 p, 2 p+1,-2 p ; 1,-4 p ; 1] .
$$


Surprisingly, the series is not summable by any classical hypergeometric sum theorem (e.g. Dixon's theorem, Watson's theorem) because the series ${ }_{3} F_{2}[-2 p$, $2 p+1,-2 p ; 1,-4 p ; z], p$ not an integer, does not converge when $z=1$; see [21, Chapter 2]. For this reason, we have to apply Zeilberger's algorithm for partial sums in order to sum the series. In the language of WZ-theory, $\bar{F}, G$ is a WZpair, where $\bar{F}(p, k):=(-1)^{p} \frac{(p !)^{3} F(p, k)}{(3 p) !}$, and $R(p, k)$ is the corresponding WZ-proof certificate.

\section{ACKNOWLEDGEMEnTS}

Most of this work was completed during a visit of the first author to TU München in July 2010. Both authors would like to thank Gregor Kemper for making this visit possible. We also want to thank the anonymous referee of this paper for valuable comments.

\section{REFERENCES}

[1] Leonid Bedratyuk. On complete system of invariants for the binary form of degree 7 . J. Symbolic Comput., 42(10):935-947, 2007. MR2361672 (2008h:13009)

[2] Leonid Bedratyuk. A complete minimal system of covariants for the binary form of degree 7 . J. Symbolic Comput., 44(2):211-220, 2009. MR2479299 (2009j:13006)

[3] Leonid Bedratyuk. Kernels of derivations of polynomial rings and Casimir elements. Ukrainian Mathematical Journal, 62(4):495-517, 2010.

[4] A Cayley. The Collected Mathematical Papers, vol 1. Cambridge University Press, Cambridge, England, 1889.

[5] Harm Derksen and Gregor Kemper. Computational invariant theory. Invariant Theory and Algebraic Transformation Groups, I. Encyclopaedia of Mathematical Sciences, 130, SpringerVerlag, Berlin, 2002. MR1918599 (2003g:13004)

[6] M. Domokos. Typical separating invariants. Transformation Groups, 12(1):49-63, 2007. MR2308028 (2007k:13008)

[7] Jan Draisma, Gregor Kemper, and David Wehlau. Polarization of separating invariants. Canad. J. Math., 60(3):556-571, 2008. MR2414957 (2009c:13011)

[8] Emilie Dufresne and Martin Kohls. A finite separating set for Daigle and Freudenburg's counterexample to Hilbert's fourteenth problem. Comm. Algebra, 38(11):3987-3992, 2010. MR2764845

[9] Jonathan Elmer. On the depth of separating algebras for finite groups. Contributions to Algebra and Geometry, to appear, 2010.

[10] Peter Fleischmann. The Noether bound in invariant theory of finite groups. Adv. Math., 156(1):23-32, 2000. MR.1800251 (2001k:13009)

[11] John Fogarty. On Noether's bound for polynomial invariants of a finite group. Electron. Res. Announc. Amer. Math. Soc., 7:5-7 (electronic), 2001. MR.1826990(2002a:13002)

[12] Gene Freudenburg. Algebraic theory of locally nilpotent derivations, volume 136 of Encyclopaedia of Mathematical Sciences. Invariant Theory and Algebraic Transformation Groups, VII. Springer-Verlag, Berlin, 2006. MR2259515 (2008f:13049)

[13] P. Gordan. Beweiss, dass jede Covariante und Invariante einer binären Form eine ganz Funktion mit numerischen Coefficienten einer endlichen Anzahl solher Formen ist. J. Reine. Angew. Math., 69:323-354, 1868.

[14] David Hilbert. Theory of algebraic invariants. Cambridge University Press, Cambridge, 1993. Translated from the German and with a preface by Reinhard C. Laubenbacher, edited and with an introduction by Bernd Sturmfels. MR 1266168 (97j:01049)

[15] Gregor Kemper. Computing invariants of reductive groups in positive characteristic. Transformation Groups, 8(2):159-176, 2003. MR.1976458 (2004b:13006)

[16] Martin Kohls and Hanspeter Kraft. Degree bounds for separating invariants. Math. Res. Lett., 17(6):1171-1182, 2010. MR2729640

[17] Peter J. Olver. Classical invariant theory, volume 44 of London Mathematical Society Student Texts. Cambridge University Press, Cambridge, 1999. MR.1694364 (2001g:13009) 
[18] Marko Petkovšek, Herbert S. Wilf, and Doron Zeilberger. $A=B$. A K Peters Ltd., Wellesley, MA, 1996. With a foreword by Donald E. Knuth, with a separately available computer disk. MR.1379802 (97j:05001)

[19] Michael Roberts. On the covariants of a binary quantic of the $n^{\text {th }}$ degree. The Quarterly Journal of Pure and Applied Mathematics, 4:168-178, 1861.

[20] Müfit Sezer. Constructing modular separating invariants. J. Algebra, 322(11):4099-4104, 2009. MR2556140 (2010i:13007)

[21] Lucy Joan Slater. Generalized hypergeometric functions. Cambridge University Press, Cambridge, 1966. MR0201688(34:1570)

[22] Lin Tan. An algorithm for explicit generators of the invariants of the basic $G_{a}$-actions. Comm. Algebra, 17(3):565-572, 1989. MR981471 (90j:13018)

[23] Arno van den Essen. An algorithm to compute the invariant ring of a $\mathbf{G}_{a}$-action on an affine variety. J. Symbolic Comput., 16(6):551-555, 1993. MR.1279532 (95c:14064)

[24] R. Weitzenböck. Über die Invarianten von linearen Gruppen. Acta. Math., 58:231-293, 1932. MR.1555349

[25] Herbert S. Wilf and Doron Zeilberger. An algorithmic proof theory for hypergeometric (ordinary and " $q$ ") multisum/integral identities. Invent. Math., 108(3):575-633, 1992. MR.1163239 (93k:33010)

Department of Mathematics, University of Bristol, University Walk, Bristol, BS8 1TW UNITED KINGDOM

E-mail address: j.elmer@bris.ac.uk

Technische Universität München, Zentrum Mathematik-M11, Boltzmannstrasse 3, 85748 Garching, Germany

E-mail address: kohls@ma.tum.de 